\title{
Phosphorylated Neurofilament Heavy Chain Correlations to Visual Function, Optical Coherence Tomography, and Treatment
}

\author{
Joshua Pasol, ${ }^{1}$ William Feuer, ${ }^{1}$ Cui Yang, ${ }^{2}$ Gerry Shaw, ${ }^{2}$ Randy Kardon, ${ }^{3}$ and John Guy \\ ${ }^{1}$ Bascom Palmer Eye Institute, Miller School of Medicine, University of Miami, 1638 NW 10th Avenue, Miami, Fl 33136, USA \\ ${ }^{2}$ Department of Neuroscience, McKnight Brain Institute, College of Medicine, University of Florida, Gainesville, FL 32610-0015, USA \\ ${ }^{3}$ Department of Ophthalmology and Visual Sciences, the University of Iowa and Veterans Hospital, Iowa City, IA 52242, USA
}

Correspondence should be addressed to John Guy, jguy@med.miami.edu

Received 24 August 2010; Revised 22 November 2010; Accepted 14 December 2010

Academic Editor: Axel Petzold

Copyright ( $) 2010$ Joshua Pasol et al. This is an open access article distributed under the Creative Commons Attribution License, which permits unrestricted use, distribution, and reproduction in any medium, provided the original work is properly cited.

\begin{abstract}
Objective. To correlate visual and neurologic clinical scores and treatment of optic neuritis and multiple sclerosis (MS) patients with assays of serum phosphorylated neurofilament heavy chain (pNF-H) and optical coherence tomography (OCT) measurements of axonal loss. Design/Methods. The Optic Neuritis Treatment Trial (ONTT) randomized 457 patients with acute optic neuritis to intravenous methylprednisolone (IVMP) followed by oral prednisone, oral prednisone or placebo treatment arms. We quantified serum pNF-H levels in 175 ONTT patients 5 years after study entry. We performed OCT measurements of macular volume and the retinal nerve fiber layer (RNFL) in a subset of 51 patients at year 15. Results. Elevated pNF-H levels at year 5 correlated to poorer visual function at study entry. Lower 15 year macular volumes and RNFL thickness correlated better with follow-up than with baseline visual function measures. With IVMP treatment, 15 year RNFL differences of the fellow eye (FE) minus the affected eye (SE) RNFLFEmSE correlated with five-year pNF-H levels. PNF-H was reduced by half with IVMP relative to placebo or by $40 \%$ relative to prednisone. Conclusions/Relevance. Acute optic neuritis patients who have more severe visual loss during initial presentation have a higher incidence of axonal loss that was slightly suppressed with IVMP treatment.
\end{abstract}

\section{Introduction}

The Optic Neuritis Treatment Trial (ONTT) compared three modes of treatment for acute unilateral optic neuritis: placebo, oral prednisone, or high-dose IVMP followed by oral prednisone [1]. Enrollment of 457 patients by 15 centers started in 1988 and was completed 3 years later. Results of the study showed that visual acuity recovered 2 weeks faster with IVMP. Six months after treatment, no difference in the generally good visual outcome was detected between the 3 treatment groups. Similarly, there were no differences in the number of a minority of patients with a poor visual outcome varying between $5 \%$ and $6 \%$ for each group and defined by the study as $20 / 50$ or worse.

Axonal and neuronal loss are increasingly recognized as the primary factors contributing to persistent deficits and disability in multiple sclerosis (MS) and optic neuritis [2-4], as also revealed by optical coherence tomography (OCT) $[5,6]$. Neurofilaments are components of the axonal cytoskeleton that consists of several subunits, including a light, medium, and heavy chain (NF-L, NF-M and NF$\mathrm{H})$. They are released into the blood stream and CSF with axonal disruption [7] that is believed to be mediated by inflammatory cells in multiple sclerosis [8]. The heavily phosphorylated axonal form of NF-H, called pNF-H, is resistant to proteases and relatively easy to detect $[9,10]$. Petzold and coworkers have described elevated levels of pNF$\mathrm{H}$ during the acute phase of visual loss from optic neuritis [11-13]. NF-H levels correlated with poor visual recovery and the development of MS. We wondered whether serum pNF-H levels may be a useful gauge of axonal loss in a large cohort of optic neuritis and MS patients.

\section{Methods}

2.1. Clinical Assessments. ONTT patients underwent visual acuity, visual field, and contrast sensitivity testing at study entry then at the one-month, 6-month, 5-year, 10-year, and 15 -year follow-up visits. EDSS assessments were performed at the 5-year, 10-year, and 15-year follow-up examinations. 
MRI was performed at baseline and at the five-year examination [14-17].

2.2. pNF-H Assay. Serum derived from venous blood was drawn during follow-up examinations 5 years after patient enrollment into the treatment arm of the study. Serum was stored in a -80 freezer for 10 years. Serum was assayed for the presence of phosphorylated neurofilament heavy chain (pNF-H), using a recently described monoclonal antibodybased assay [18]. Wells of microtitre plates were coated overnight with $100 \mu \mathrm{L}$ of purified pNF-H monoclonal capture antibody clone $\mathrm{AH} 1$, diluted in $10 \mathrm{~mL} 0.05 \mathrm{M}$ carbonate buffer, $\mathrm{pH} 9.5$ to give a final concentration of $1 \mu \mathrm{g} \mathrm{IgG}$ per ml. The antibody and carbonate mix was decanted and the plates blocked with $150 \mu \mathrm{L}$ of $5 \%$ nonfat milk in TBS for 1 hour. The plate was washed with $2 \%$ nonfat milk in TBS and $0.1 \%$ Tween 20 (pH 7.5). After washing, a total of $50 \mu \mathrm{L}$ of standard or $20 \mu \mathrm{L}$ serum sample plus $30 \mu \mathrm{L} 2 \%$ nonfat milk in TBS and $0.1 \%$ Tween 20 were added in duplicate to the plate. The plates are incubated on a shaker at room temperature for $1 \mathrm{~h}$. After washing, $100 \mu \mathrm{L}$ of purified mouse anti-pNF-H monoclonal antibody, directly coupled to horse radish peroxidase (HRP) at a final concentration of $1 \mu \mathrm{g} / \mathrm{mL}$ in $2 \%$ nonfat milk in TBS plus $0.1 \%$ Tween 20 were added to each well and the plate incubated for $1 \mathrm{~h}$ at RT. After a final wash, the reaction was visualized using $100 \mu \mathrm{L}$ per well of $3,3^{\prime}, 5,5^{\prime}$-tetramethybenzidine HRP developer solution (Thermo Fisher, Rockford, IL). Absorbance was read at a wavelength of $450 \mathrm{~nm}$ on a Tecan SpectraFluor ELISA plate reader, 15 minutes after addition of chromogen, and after stopping the reaction by the addition of $50 \mu \mathrm{L}$ $1 \mathrm{M} \mathrm{H}_{2} \mathrm{SO}_{4}$ per well. The entire set of samples were run as described twice with excellent reproducibility. The pNF-H antibodies used here can be obtained commercially from EnCor Biotechnology Inc. (Gainesville, FL).

2.3. OCT. Optical coherence tomography (OCT) $[19,20]$, an indirect way of measuring retinal nerve fiber layer thickness around the optic nerve, and macular volumes were obtained in a subset of 51 patients, 10 years after drawing blood samples (15 years after ONTT enrollment). Peripapillary retinal nerve fiber layer (RNFL) thickness was evaluated using the fast RNFL program of the Stratus OCT (Carl Zeiss Meditec, Dublin, CA), and analyzed using software version 3.0. RNFL thickness is determined at 256 points around a circular scan (diameter $3.4 \mathrm{~mm}$ ) around the center of the optic disc that is repeated 3 consecutive times. For each eye, RNFL scans were repeated 4 times, exported on an electronic worksheet, and an average scan was computed. RNFL thickness and macular volume were evaluated from the average scan. OCT data from the clinical centers was collected by the reading center at the University of Iowa.

2.4. Data Analysis. Pearson correlation analysis of data was performed using SPSS statistical software (Chicago, IL). The association of subject characteristics to pNF-H levels was studied with multiple regression. The pNF-H data were square root transformed to effect normality of
TABLE 1: Baseline (recruitment) participant characteristics of ONTT subjects undergoing pNF-H testing.

\begin{tabular}{lc}
\hline Number & 175 \\
Mean (SD) [range] age & $33.2(6.8)[18,46]$ \\
$N(\%)$ Female & $140(80 \%)$ \\
$N$ (\%) Race/Ethnicity & \\
$\quad$ Caucasian & $159(91 \%)$ \\
$\quad$ African-American & $15(9 \%)$ \\
$\quad$ Hispanic & $1(1 \%)$ \\
$N$ (\%) Right eye affected & $92(53 \%)$ \\
$N$ (\%) Initial multiple sclerosis & \\
$\quad$ None & $114(65 \%)$ \\
$\quad$ Possible & $35(20 \%)$ \\
$\quad$ Probable & $9(5 \%)$ \\
$\quad$ Definite & $17(10 \%)$ \\
Treatment group & \\
$\quad$ IVMP & $63(36 \%)$ \\
$\quad$ Prednisone & $63(36 \%)$ \\
$\quad$ Placebo & $49(28 \%)$ \\
\hline
\end{tabular}

distribution of residuals. Relationships between the square root of pNF-H were investigated within groups with Pearson correlation and the two-sample $t$-test. Linear regression was used to assess the difference between groups and whether the relationship was different between clinically isolated optic neuritis patients and those with clinically definite MS. Analysis of covariance was used to assess the influence of treatment on pNF-H levels.

\section{Results}

3.1. $\mathrm{pNF}-\mathrm{H}$ and Treatment. We quantified pNF-H levels in the serum of $175(44 \%)$ of the 397 patients, $87 \%$ of those initially entered into the ONTT, who returned for follow-up examinations 5 years after enrollment into the study. Their baseline characteristics are listed in Table 1. A normal control group of age-matched volunteers free of any neurologic diseases had a mean pNF-H concentration of $0.07 \pm 0.05$ nanograms $(\mathrm{ng})$ per milliliter $(\mathrm{mL})$ (mean \pm standard deviation) $(n=12)$. Sixty-three of the ONTT patients had received IVMP, 63 patients had received oral prednisone, and 49 patients had received oral placebo. Post hoc least significant difference tests revealed serum pNF-H was reduced by $50 \%$ with IVMP with a value of $0.096 \pm$ $0.692 \mathrm{ng}$ per $\mathrm{mL}$ relative to placebo with a mean of $0.192 \pm$ $0.399 \mathrm{ng}$ per $\mathrm{mL}$ (difference $=-0.067 ; P=.047$ ) or by $40 \%$ relative to prednisone with a mean of $0.145 \pm 0.134 \mathrm{ng}$ per $\mathrm{mL}($ difference $=-0.073, P=.021)($ Figure $1(\mathrm{a}))$.

3.2. pNF-H and Clinical Parameters. Next, we investigated whether the increases in pNF-H levels of placebo and prednisone-treated patients relative to those who received IVMP, shown by the study to reduce the risk of developing MS for approximately 2 years [21], were due to optic neuritis itself or the coexistence of MS. For this analysis, we separated 


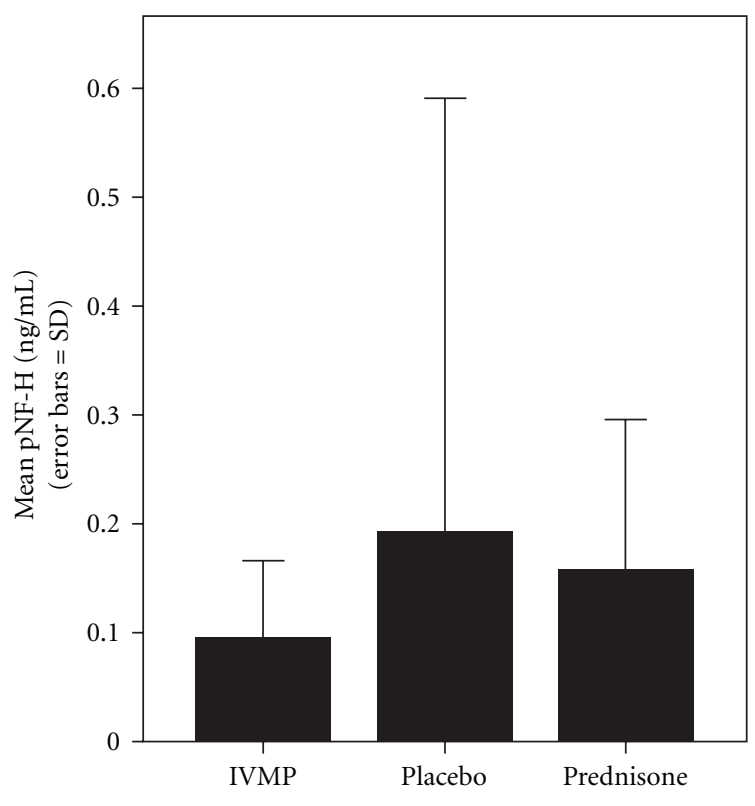

(a)

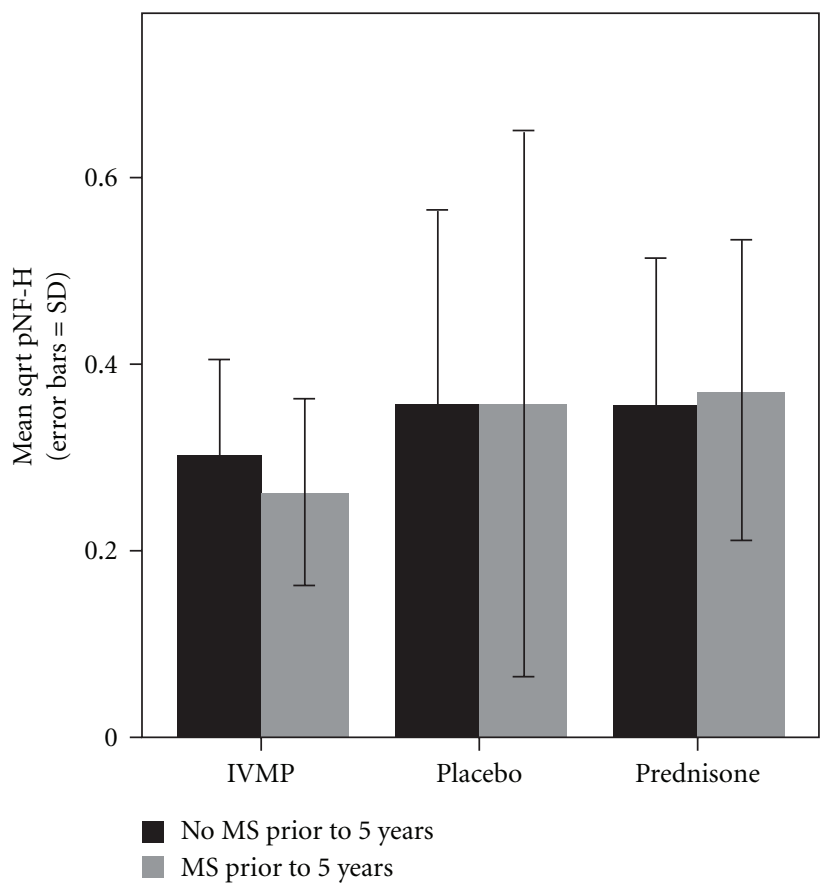

(b)

Figure 1: Bar plots of pNF-H concentration (nanograms per $\mathrm{mL}$ ) in optic neuritis patients by treatment group (a) and between optic neuritis cases with or without MS at 5 years (b). Error bars = standard deviation.

treatment groups into those with clinically isolated optic neuritis and those with multiple sclerosis (Figure 1(b)). Among these 175 patients, 17 (10\%) initially had clinically definite multiple sclerosis (CDMS). During ONTT followup, another $78(46 \%)$ developed CDMS after an average of 4.1 years. We found no significant difference $(P=.74$, ANOVA) in average pNF-H concentration between patients with onset of CDMS at or prior to the year-five visit (mean $\pm \mathrm{SD}=0.16 \pm 0.31$ ), patients with CDMS after the fiveyear visit $(0.11 \pm 0.11)$, or optic neuritis patients who never developed CDMS (mean $\pm \mathrm{SD}=0.14 \pm 0.16$ ). Neither was pNF-H concentration predictive of onset of CDMS after five years among the 98 patients who had not previously developed it $(P=.51$, Cox proportional hazards regression. Risk Ratio $=0.86,95 \%$ CI: $0.54,1.36$ for a 0.1 unit higher concentration of pNF-H). Thus, reductions in pNF-H by IVMP were not due to suppression of MS, but likely due to suppression of axonal injury in the optic nerve.

Next, we compared baseline and 5-year measurements by randomized treatment assignment and 15-year followup with OCT (Table 2). For the subset of 51 patients that had underwent OCTs during the year- 15 visit we found that higher pNF-H levels at year 5 correlated to poorer visual acuity $(P=.001)$, worse contrast sensitivity $(P=.007)$, and denser visual field defects $(P=.038)$ at the baseline (BL) study entry visit. Figure 2(a) illustrates the increase in serum pNF-H with worsening baseline visual acuity in the affected eyes with acute optic neuritis. Figure 2(b) shows that higher pNF-H correlates with more severe visual field defects at baseline. Figure 2(c) shows higher serum pNF-H that correlates with loss of contrast sensitivity at entry into the ONTT. No correlations of visual function to pNF-H were seen in any of the follow-up visits. No correlations of pNF$\mathrm{H}$ to EDSS scores only done at year 5 , year ten, and year 15 were detected. No correlations of pNF-H to MRI lesions were detected. Table 3 shows the progression of MS EDSS from year 5 to 15 for those diagnosed with MS at baseline, 6 months, 5 years, 10 years, or 15 years after study entry. Interestingly, the incidence of diagnosed MS was significantly lower in the cases that returned for the 15-year follow-up for OCT $(P=.012)$. We discuss their OCT findings next.

3.3. OCT. OCT signal strengths ranged from 5 to 10 in both fellow and affected eyes, with $44(86 \%)$ and 47 (92\%) having signal strength greater or equal to 7 . Table 4 shows the RNFL and macular volumes by treatment group. Differences between affected eyes relative to the fellow eyes were statistically significant.

3.4. OCT and Clinical Parameters. We found a significant correlation of baseline visual acuity loss to a reduction in total macular volume in the subset of 51 patients that had their RNFL and macular volumes thickness measured with Stratus OCT at the 15-year follow-up visit. Table 5 shows the OCT correlations to visual function and EDSS for each visit alongside the pNF-H measurements. Macular volume and RNFL loss correlated to a significant loss of visual acuity at the one-month follow-up visit. Contrast sensitivity loss of affected eyes (CSENAF) and denser visual 


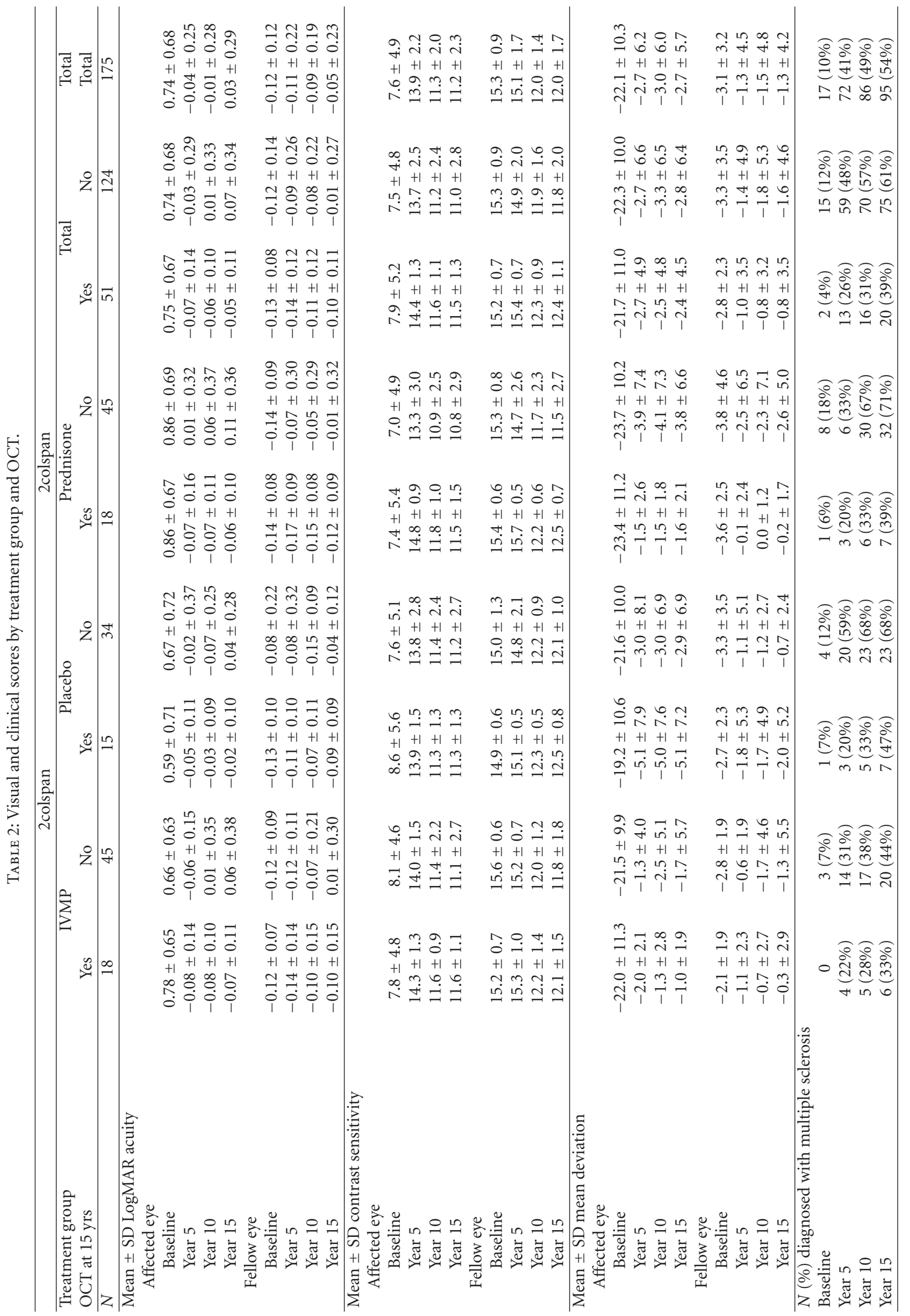


TABLE 3: MS characteristics.

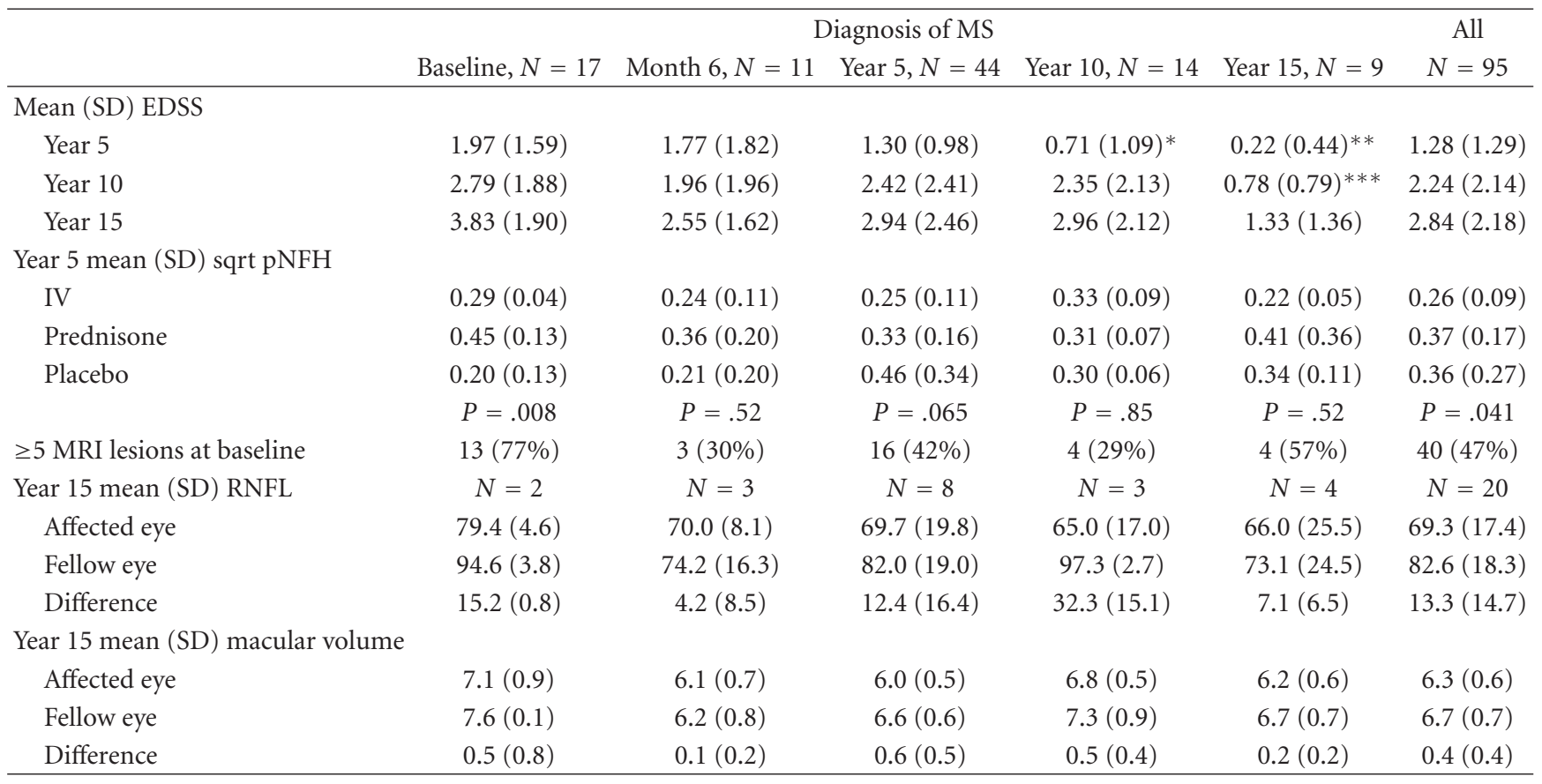

TABLE 4: OCT data RNFL and macular volumes.

\begin{tabular}{|c|c|c|c|c|}
\hline & $\begin{array}{c}\text { IVMP } \\
N=18\end{array}$ & $\begin{array}{l}\text { Placebo } \\
N=15\end{array}$ & $\begin{array}{c}\text { Prednisone } \\
N=18\end{array}$ & $\begin{array}{c}\text { Total } \\
N=51\end{array}$ \\
\hline \multicolumn{5}{|l|}{ Signal strength } \\
\hline Affected eye & $8.2 \pm 1.2$ & $8.2 \pm 1.2$ & $7.9 \pm 1.5$ & $8.1 \pm 1.3$ \\
\hline Fellow eye & $8.1 \pm 1.8$ & $8.5 \pm 1.1$ & $8.4 \pm 1.2$ & $8.3 \pm 1.4$ \\
\hline \multicolumn{5}{|c|}{ Year 15 mean (SD) RNFL } \\
\hline Affected eye & $80.9 \pm 18.1$ & $71.3 \pm 22.7$ & $78.2 \pm 17.1$ & $77.1 \pm 19.3$ \\
\hline Fellow eye & $95.2 \pm 16.7$ & $87.1 \pm 18.1$ & $94.5 \pm 18.5$ & $92.6 \pm 17.8$ \\
\hline Difference (AF-FE) & $-14.3 \pm 17.3$ & $-15.8 \pm 22.4$ & $-16.3 \pm 15.9$ & $-15.5 \pm 18.2$ \\
\hline$P$-value & .003 & .016 & $<.001$ & $<.001$ \\
\hline \multicolumn{5}{|c|}{ Year 15 mean (SD) macular volume } \\
\hline Affected eye & $6.3 \pm 0.5$ & $6.5 \pm 0.7$ & $6.4 \pm 0.5$ & $6.4 \pm 0.6$ \\
\hline Fellow eye & $6.7 \pm 0.5$ & $6.9 \pm 0.8$ & $7.0 \pm 0.6$ & $6.9 \pm 0.7$ \\
\hline Difference & $-0.4 \pm 0.5$ & $-0.4 \pm 0.5$ & $-0.7 \pm 0.6$ & $-0.5 \pm 0.6$ \\
\hline$P$-value & .005 & .010 & $<.001$ & $<.001$ \\
\hline
\end{tabular}

field defects (MDSE) correlated to the OCT parameters of axonal loss at almost every time point except the baseline clinical examination.

Looking at the OCT findings by treatment group, we found no significant difference of mean IVMP RNFL = $81 \mu \mathrm{m}(n=18), 78 \mu \mathrm{m}$ with prednisone $(n=18)$, or $71 \mathrm{~mm}$ placebo $(n=15)$ (Figure $3(\mathrm{a}))$. RNFL measurements of affected and nonaffected eyes of patients with CDMS ( $n=20$ ), respectively, $69 \mu \mathrm{m}$ and $82 \mu \mathrm{m}$, were significantly different (resp., $P=.018$ and $P=.001$ ) from those with clinically isolated optic neuritis, respectively, $83 \mu \mathrm{m}$ and $99 \mu \mathrm{m}(n=31)$. The average \pm SD RNFL thickness in all affected eyes of $77.1 \pm 19.3 \mu \mathrm{m}$ was significantly thinner than the fellow eye $(P<.001, t$-test $)$ with an average $92.6 \pm$ $17.8 \mu \mathrm{m}$. These differences were also true for the macular volumes.

Lastly, we found no statistical correlation of pNF-H levels to recovery of visual acuity at 6 months or RNFL thickness at 15 years in patients with or without MS. However, RNFL thickness differences of fellow eye (FE) minus study eye (SE) RNFLFEmSE correlated to pNF-H levels in the IV methylprednisolone group $(P=.04)$ (Figure 3(b)). Thus, IVMP-treated patients with greater loss of axons at year 15 had increased pNF-H levels at year 5 . 
TABLE 5: Correlations of clinical profile to OCT and pNF-H.

\begin{tabular}{|c|c|c|c|c|c|}
\hline Measurement & Visit & Statistic & $\begin{array}{c}\text { Study eye RNFL } \\
\text { thickness }\end{array}$ & $\begin{array}{l}\text { Study eye total } \\
\text { macular volume }\end{array}$ & $\begin{array}{c}\text { Square root } \\
\text { pNFH }\end{array}$ \\
\hline \multirow{9}{*}{ EDSS } & \multirow{3}{*}{ Year 5} & $r$ & -0.28 & -0.28 & 0.15 \\
\hline & & $P$-value & .048 & .057 & .291 \\
\hline & & $N$ & 50 & 47 & 50 \\
\hline & \multirow{3}{*}{ Year 10} & $r$ & -0.38 & -0.11 & 0.02 \\
\hline & & $P$-value & .008 & .473 & .901 \\
\hline & & $N$ & 49 & 46 & 49 \\
\hline & \multirow{3}{*}{ Year 15} & $r$ & -0.23 & 0.07 & 0.03 \\
\hline & & $P$-value & .107 & .641 & .841 \\
\hline & & $N$ & 51 & 48 & 51 \\
\hline \multirow{18}{*}{ LogMAR study eye acuity } & \multirow{3}{*}{ Baseline } & $r$ & -0.09 & -0.29 & 0.44 \\
\hline & & $P$-value & .512 & .046 & .001 \\
\hline & & $N$ & 51 & 48 & 51 \\
\hline & \multirow{3}{*}{ Month 1} & $r$ & -0.44 & -0.41 & 0.13 \\
\hline & & $P$-value & .002 & .005 & .364 \\
\hline & & $N$ & 48 & 45 & 48 \\
\hline & \multirow{3}{*}{ Month 6} & $r$ & -0.35 & -0.22 & -0.11 \\
\hline & & $P$-value & .011 & .132 & .442 \\
\hline & & $N$ & 51 & 48 & 51 \\
\hline & \multirow{3}{*}{ Year 5} & $r$ & -0.27 & -0.26 & -0.18 \\
\hline & & $P$-value & .060 & .074 & .206 \\
\hline & & $N$ & 51 & 48 & 51 \\
\hline & \multirow{3}{*}{ Year 10} & $r$ & -0.52 & -0.30 & -0.08 \\
\hline & & $P$-value & $<.001$ & .040 & .565 \\
\hline & & $N$ & 50 & 47 & 50 \\
\hline & \multirow{3}{*}{ Year 15} & $r$ & -0.48 & -0.36 & 0.00 \\
\hline & & $P$-value & $<.001$ & .011 & .974 \\
\hline & & $N$ & 51 & 48 & 51 \\
\hline \multirow{18}{*}{ Sensitivity study eye contrast } & \multirow{3}{*}{ Baseline } & $r$ & 0.18 & 0.23 & -0.37 \\
\hline & & $P$-value & .207 & .121 & .007 \\
\hline & & $N$ & 51 & 48 & 51 \\
\hline & \multirow{3}{*}{ Month 1} & $r$ & 0.54 & 0.42 & -0.17 \\
\hline & & $P$-value & $<.001$ & .004 & .256 \\
\hline & & $N$ & 48 & 45 & 48 \\
\hline & \multirow{3}{*}{ Month 6} & $r$ & 0.54 & 0.44 & -0.12 \\
\hline & & $P$-value & $<.001$ & .002 & .419 \\
\hline & & $N$ & 51 & 48 & 51 \\
\hline & \multirow{3}{*}{ Year 5} & $r$ & 0.45 & 0.37 & -0.04 \\
\hline & & $P$-value & .001 & .010 & .759 \\
\hline & & $N$ & 50 & 47 & 50 \\
\hline & \multirow{3}{*}{ Year 10} & $r$ & 0.52 & 0.43 & 0.01 \\
\hline & & $P$-value & $<.001$ & .003 & .964 \\
\hline & & $N$ & 49 & 46 & 49 \\
\hline & \multirow{3}{*}{ Year 15} & $r$ & 0.58 & 0.52 & -0.06 \\
\hline & & $P$-value & $<.001$ & $<.001$ & .685 \\
\hline & & $N$ & 51 & 48 & 51 \\
\hline
\end{tabular}


Table 5: Continued.

\begin{tabular}{|c|c|c|c|c|c|}
\hline Measurement & Visit & Statistic & $\begin{array}{l}\text { Study eye RNFL } \\
\text { thickness }\end{array}$ & $\begin{array}{l}\text { Study eye total } \\
\text { macular volume }\end{array}$ & $\begin{array}{c}\text { Square root } \\
\text { pNFH }\end{array}$ \\
\hline \multirow{18}{*}{ Study eye visual field mean deviation } & \multirow{3}{*}{ Baseline } & $r$ & 0.24 & 0.30 & -0.29 \\
\hline & & $P$-value & .095 & .036 & .038 \\
\hline & & $N$ & 51 & 48 & 51 \\
\hline & \multirow{3}{*}{ Month 1} & $r$ & 0.56 & 0.46 & -0.11 \\
\hline & & $P$-value & $<.001$ & .001 & .462 \\
\hline & & $N$ & 48 & 45 & 48 \\
\hline & \multirow{3}{*}{ Month 6} & $r$ & 0.55 & 0.34 & 0.08 \\
\hline & & $P$-value & $<.001$ & .019 & .593 \\
\hline & & $N$ & 51 & 48 & 51 \\
\hline & \multirow{3}{*}{ Year 5} & $r$ & 0.48 & 0.32 & 0.06 \\
\hline & & $P$-value & $<.001$ & .029 & .650 \\
\hline & & $N$ & 51 & 48 & 51 \\
\hline & \multirow{3}{*}{ Year 10} & $r$ & 0.57 & 0.40 & 0.10 \\
\hline & & $P$-value & $<.001$ & .006 & .512 \\
\hline & & $N$ & 50 & 47 & 50 \\
\hline & \multirow{3}{*}{ Year 15} & $r$ & 0.55 & 0.30 & 0.05 \\
\hline & & $P$-value & $<.001$ & .041 & .730 \\
\hline & & $N$ & 51 & 48 & 51 \\
\hline
\end{tabular}

\section{Discussion}

We have shown here that serum pNF-H levels measured 5 years after acute optic neuritis correlate with baseline visual function. Next, early follow-up visual function correlates with RNFL and macular volumes measured at 15 years. Lastly, pNF-H levels at year 5 do not correlate with RNFL and macular volumes in the affected eye, but they do correlate with the difference between study and fellow eyes at least in the IVMP group.

Our findings of increased pNF-H levels with more severe baseline visual deficits are consistent with axonal transection by inflammatory cells that is believed to contribute to the loss of axons [8]. This insult occurs at baseline, and the axons start the process of dying off, releasing pNF-H into the serum as they do. This process of degeneration appears to continue for at least 5 years. OCT measures what is remaining after the axons have died off, thus a better correlation to the follow-up exams. Teunissen et al. showed that neurofilament heavy chain levels detected in the CSF of patients with MS and clinically isolated syndrome were higher than normal controls, reaching their highest levels during acute exacerbations of disease activity [22]. Petzold et al., who obtained serum samples for measurements of the pNF-H during the acute phases of optic neuritis, found higher levels in those who had a poor visual recovery [12]. Since acute serum samples from ONTT patients were unavailable for inclusion in our study here, we had only a single snapshot of $\mathrm{pNF}-\mathrm{H}$, at year 5. Therefore, we are unable to resolve whether axonal transection by inflammatory cells was suppressed by IVMP or whether treatment induced a decrease in release of pNF-H. Still, due to its high resistance to protease digestion [9], pNF-H may have persisted at higher levels for years, though this seems unlikely. Alternately, a small amount of axonal loss and regeneration may be an ongoing feature of optic neuropathy, and IVMP may have a long-term effect by reducing this.

While we found no correlation of pNF-H to clinical measures of visual function at the very late time points, and pNF-H was not associated with study eye RNFL even in the IVMP group, the difference between fellow eye and study eye RNFL thickness correlated with pNF-H levels in the IVMP group. This finding validates $\mathrm{pNF}-\mathrm{H}$ as a biomarker of suppression of axonal loss by high-dose methylprednisolone, now standard therapy for acute optic neuritis and MS exacerbations. Since no difference in serum pNF-H concentration between clinically isolated optic neuritis and MS patients were detected, it is unlikely that brain lesions outside the optic nerve contributed to the differences in pNF-H between the IV methylprednisolone and the other two treatment groups. We also excluded brain lesions in clinically isolated optic neuritis and multiple sclerosis patients detected on T2-weighted MRI as a cause for the differences in pNF-H between the treatment groups. 


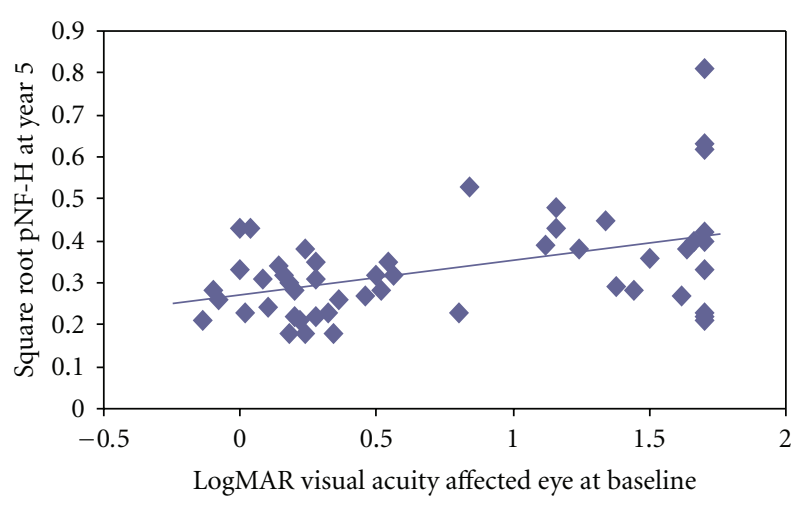

(a)

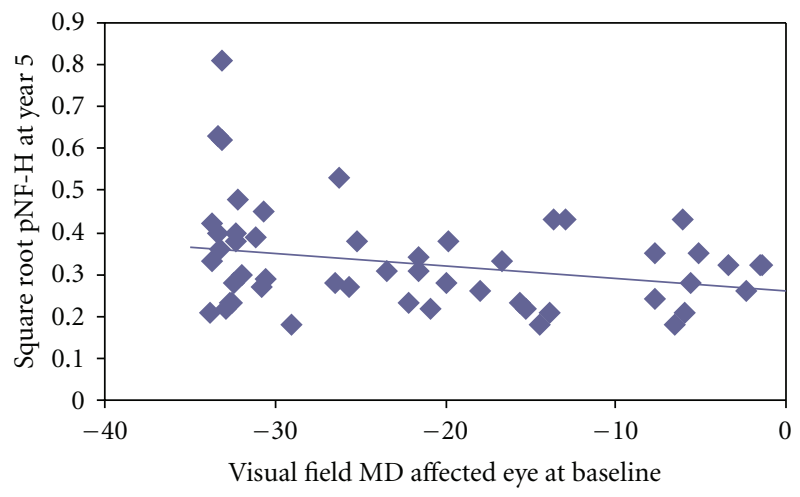

(b)

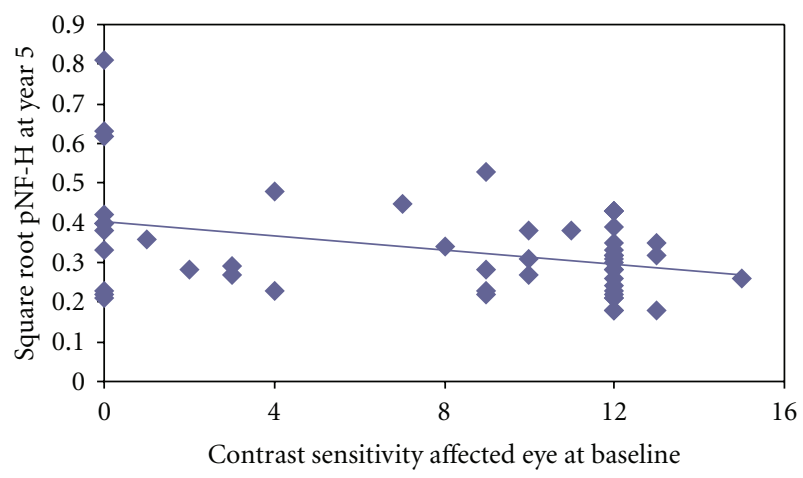

(c)

FIgURE 2: (a) A scatterplot shows the increasing pNF-H with worsening baseline visual acuity in the affected eyes with acute optic neuritis. (b) The scatterplot shows elevated serum pNF-H correlates with poorer contrast sensitivity at entry into the ONTT. (c) A scatterplot shows that higher pNF-H correlates with more severe visual field defects at baseline.

Consistent with our observations in ONTT patients, elevation of serum pNF-H has also been found in another disease that affects the optic nerve, Lebers' hereditary optic neuropathy (LHON) [23]. Elevations in serum pNF$\mathrm{H}$ have also been detected in animals with experimental autoimmune encephalomyelitis (EAE) and suppressed with treatment [24]. As further verification that axonal loss from optic neuritis was the likely source of the pNF-H in ONTT patients, we turned to transgenic TCR MOG

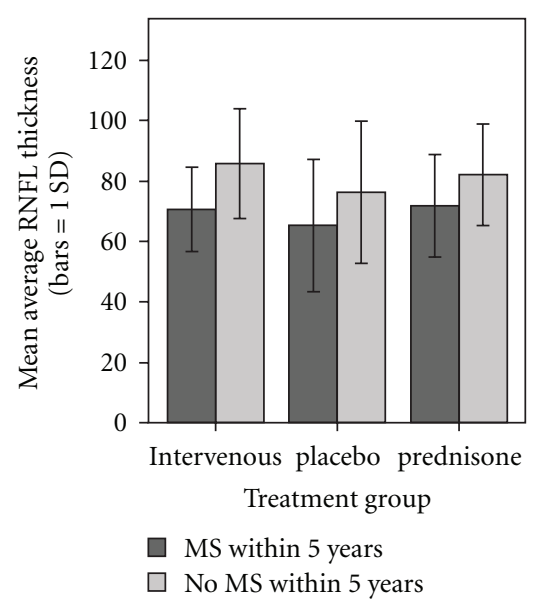

(a)

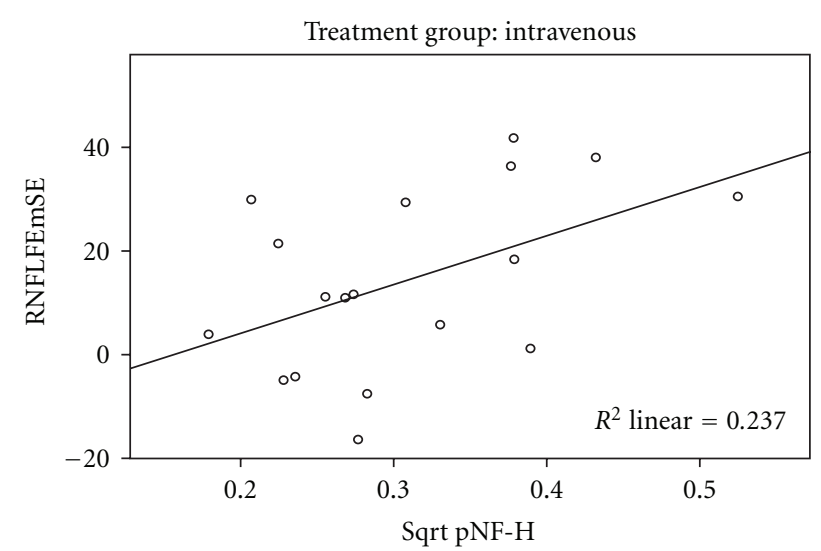

(b)

FIGURE 3: (a) A barplot of OCT RNFL thickness at 15 years in a subset of 51 patients shows no significant difference of mean RNFL with treatment of optic neuritis patients with MS or clinically isolated optic neuritis patients without MS. (b) A scatterplot of RNFL thickness difference of the fellow eye (FE) minus the study eye (SE) RNFLFEmSE correlated to serum pNF-H levels in the IVMP group.

mice that develop only optic neuritis that in turn causes loss of retinal ganglion cells and their optic nerve axons [25]. Preliminary unpublished observations in these animals revealed a several fold elevation in serum pNF-H levels relative to control littermates (presented at the AAN Toronto 2010-[P06.229]). Thus, serum pNF-H elevations in ONTT patients were likely due to optic neuritis, and this biomarker is likely a reliable monitor for suppression of axonal injury in the optic nerve.

Despite the OCT and pNF-H correlation data supporting a long-term benefit of treatment with IVMP, its effect on visual parameters and neurologic or MRI lesions was virtually nil. For month 1 , month 6 , year 5 , year 10 , and year 15 , treatment had very little affect on outcome variables of acuity, contrast sensitivity, visual field mean deviation, EDSS score, or MRI lesions [1]. Only contrast sensitivity at month 1 and month 6, and mean deviation at month 6 were, or approached being, significantly different with IVMP. Thus, 
contrast sensitivity may be a sensitive clinical measure of treatment outcome. In fact, using low contrast acuity as an outcome measure, Balcer and colleagues demonstrated that natalizumab treatment of MS patients was beneficial to this parameter of visual function only [26]. That study did not examine the effects of treatment on axonal or neuronal loss.

Corticosteroids have been shown to suppress axonal loss in the optic nerves of animals with experimental autoimmune encephalomyelitis (EAE) [27]. On the other hand, in this animal model of MS, corticosteroids have also been shown to induce neuronal degeneration in retinal ganglion cells whose axons comprise the optic nerve [28]. Thus, corticosteroids appear to have opposing effects on different elements of the visual system in animal experimental models. In optic neuritis associated with neuromyelitis optica (NMO), IVMP treatment resulted in a better visual outcome and suppressed RNFL loss [29]. In a study of MS, no differences in RNFL were detected between patients treated with interferon beta-1a, interferon beta-1b, or glatiramer acetate relative to placebo [30]. To our knowledge, our study is the first demonstrating that an anti-inflammatory pharmacologic agent is able to modulate a marker of axonal loss in optic neuritis patients that do not have NMO. Since axonal loss is a characteristic of optic neuritis that may be slightly suppressed by methylprednisolone at a dose of 1 gram a day for 3 days, one can only wonder whether substantial escalation of the dose would have a greater neuroprotective effect than that seen here. Perhaps, those patients with worse baseline visual function found to have elevated pNF-H could be targeted with a neuroprotective strategy [31] as pNF-H levels too correlated with loss of RNFL and macular volumes ten years later.

\section{Acknowledgments}

Serum samples used in the analyses were provided by the ONTT study Group. Mabel Wilson edited the paper. This Study supported by R01EY07982. Gerry Shaw holds equity in EnCor Biotechnology Inc., a company commercializing antibodies and the ELISA assay used in this study, and may benefit by receiving royalties or equity growth.

\section{References}

[1] R. W. Beck, P. A. Cleary, M. M. Anderson Jr. et al., "A randomized, controlled trial of corticosteroids in the treatment of acute optic neuritis," The New England Journal of Medicine, vol. 326, no. 9, pp. 581-588, 1992.

[2] C. Bjartmar, J. R. Wujek, and B. D. Trapp, "Axonal loss in the pathology of MS: consequences for understanding the progressive phase of the disease," Journal of the Neurological Sciences, vol. 206, no. 2, pp. 165-171, 2003.

[3] C. Bjartmar, R. P. Kinkel, G. Kidd, R. A. Rudick, and B. D. Trapp, "Axonal loss in normal-appearing white matter in a patient with acute MS," Neurology, vol. 57, no. 7, pp. 12481252, 2001.

[4] G. C. DeLuca, K. Williams, N. Evangelou, G. C. Ebers, and M. M. Esiri, "The contribution of demyelination to axonal loss in multiple sclerosis," Brain, vol. 129, no. 6, pp. 1507-1516, 2006.
[5] F. Costello, S. Coupland, W. Hodge et al., "Quantifying axonal loss after optic neuritis with optical coherence tomography," Annals of Neurology, vol. 59, no. 6, pp. 963-969, 2006.

[6] S. A. Trip, P. G. Schlottmann, S. J. Jones et al., "Retinal nerve fiber layer axonal loss and visual dysfunction in optic neuritis," Annals of Neurology, vol. 58, no. 3, pp. 383-391, 2005.

[7] A. Petzold, D. Baker, G. Pryce, G. Keir, E. J. Thompson, and G. Giovannoni, "Quantification of neurodegeneration by measurement of brain-specific proteins," Journal of Neuroimmunology, vol. 138, no. 1-2, pp. 45-48, 2003.

[8] B. D. Trapp, J. Peterson, R. M. Ransohoff, R. Rudick, S. Mörk, and L. Bö, "Axonal transection in the lesions of multiple sclerosis," The New England Journal of Medicine, vol. 338, no. 5, pp. 278-285, 1998.

[9] G. Shaw, C. Yang, R. Ellis et al., "Hyperphosphorylated neurofilament NF-H is a serum biomarker of axonal injury," Biochemical and Biophysical Research Communications, vol. 336, no. 4, pp. 1268-1277, 2005.

[10] A. Petzold and G. Shaw, "Comparison of two ELISA methods for measuring levels of the phosphorylated neurofilament heavy chain," Journal of Immunological Methods, vol. 319, no. 1-2, pp. 34-40, 2007.

[11] A. Petzold, "Neurofilament phosphoforms: surrogate markers for axonal injury, degeneration and loss," Journal of the Neurological Sciences, vol. 233, no. 1-2, pp. 183-198, 2005.

[12] A. Petzold, K. Rejdak, and G. T. Plant, "Axonal degenaration and inflammation in acute optic neuritis," Journal of Neurology, Neurosurgery and Psychiatry, vol. 75, no. 8, pp. 1178-1180, 2004.

[13] A. Petzold, M. J. Eikelenboom, G. Keir et al., "The new global multiple sclerosis severity score (MSSS) correlates with axonal but not glial biomarkers," Multiple Sclerosis, vol. 12, no. 3, pp. 325-328, 2006.

[14] R. L. Gal, "Visual function more than 10 years after optic neuritis: experience of the optic neuritis treatment trial," American Journal of Ophthalmology, vol. 137, no. 1, pp. 77-83, 2004.

[15] R. W. Beck, "The optic neuritis treatment trial: three-year follow-up results," Archives of ophthalmology, vol. 113, no. 2, pp. 136-137, 1995.

[16] R. W. Beck, J. D. Trobe, P. S. Moke et al., "Visual function 5 years after optic neuritis: experience of the optic neuritis treatment trial," Archives of Ophthalmology, vol. 115, no. 12, pp. 1545-1552, 1997.

[17] Optic Neuritis Study Group, "Visual function 15 years after optic neuritis: a final follow-up report from the Optic Neuritis Treatment Trial," Ophthalmology, vol. 115, no. 6, pp. 10791082, 2008.

[18] K. Boylan, C. Yang, J. Crook et al., "Immunoreactivity of the phosphorylated axonal neurofilament $\mathrm{H}$ subunit ( $\mathrm{pNF}-\mathrm{H})$ in blood of ALS model rodents and ALS patients: evaluation of blood pNF-H as a potential ALS biomarker," Journal of Neurochemistry, vol. 111, no. 5, pp. 1182-1191, 2009.

[19] D. Huang, E. A. Swanson, C. P. Lin et al., "Optical coherence tomography," Science, vol. 254, no. 5035, pp. 1178-1181, 1991.

[20] J. S. Schuman, T. Pedut-Kloizman, E. Hertzmark et al., "Reproducibility of nerve fiber layer thickness measurements using optical coherence tomography," Ophthalmology, vol. 103, no. 11, pp. 1889-1898, 1996.

[21] R. W. Beck, P. A. Cleary, J. D. Trobe et al., "The effect of corticosteroids for acute optic neuritis on the subsequent development of multiple sclerosis," The New England Journal of Medicine, vol. 329, no. 24, pp. 1764-1769, 1993. 
[22] C. E. Teunissen, E. Iacobaeus, M. Khademi et al., "Combination of CSF $\mathrm{N}$-acetylaspartate and neurofilaments in multiple sclerosis," Neurology, vol. 72, no. 15, pp. 1322-1329, 2009.

[23] J. Guy, G. Shaw, F. N. Ross-Cisneros et al., "Phosphorylated neurofilament heavy chain is a marker of neurodegeneration in Leber hereditary optic neuropathy (LHON)," Molecular Vision, vol. 14, pp. 2443-2450, 2008.

[24] M. M. Gresle, G. Shaw, B. Jarrott et al., "Validation of a novel biomarker for acute axonal injury in experimental autoimmune encephalomyelitis," Journal of Neuroscience Research, vol. 86, no. 16, pp. 3548-3555, 2008.

[25] Y. Guan, K. S. Shindler, P. Tabuena, and A. M. Rostami, "Retinal ganglion cell damage induced by spontaneous autoimmune optic neuritis in MOG-specific TCR transgenic mice," Journal of Neuroimmunology, vol. 178, no. 1-2, pp. 4048, 2006.

[26] L. J. Balcer, S. L. Galetta, P. A. Calabresi et al., "Natalizumab reduces visual loss in patients with relapsing multiple sclerosis," Neurology, vol. 68, no. 16, pp. 1299-1304, 2007.

[27] M. Dutt, P. Tabuena, E. Ventura, A. Rostami, and K. S. Shindler, "Timing of corticosteroid therapy is critical to prevent retinal ganglion cell loss in experimental optic neuritis," Investigative Ophthalmology \& Visual Science, vol. 51, no. 3, pp. 1439-1445, 2010.

[28] R. Diem, M. Hobom, K. Maier et al., "Methylprednisolone increases neuronal apoptosis during autoimmune CNS inflammation by inhibition of an endogenous neuroprotective pathway," Journal of Neuroscience, vol. 23, no. 18, pp. 69937000, 2003.

[29] M. Nakamura, T. Nakazawa, H. Doi et al., "Early highdose intravenous methylprednisolone is effective in preserving retinal nerve fiber layer thickness in patients with neuromyelitis optica," Graefe's Archive for Clinical and Experimental Ophthalmology, vol. 248, pp. 1777-1785, 2010.

[30] E. García-Martín, V. Pueyo, J. Fernández et al., "Effect of treatment in loss of retinal nerve fibre layer in multiple sclerosis patients," Archivos de la Sociedad Espanola de Oftalmologia, vol. 85, no. 6, pp. 209-214, 2010.

[31] X. Qi, A. S. Lewin, L. Sun, W. W. Hauswirth, and J. Guy, "Suppression of mitochondrial oxidative stress provides long-term neuroprotection in experimental optic neuritis," Investigative Ophthalmology \& Visual Science, vol. 48, no. 2, pp. 681-691, 2007. 


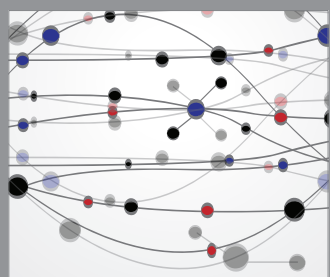

The Scientific World Journal
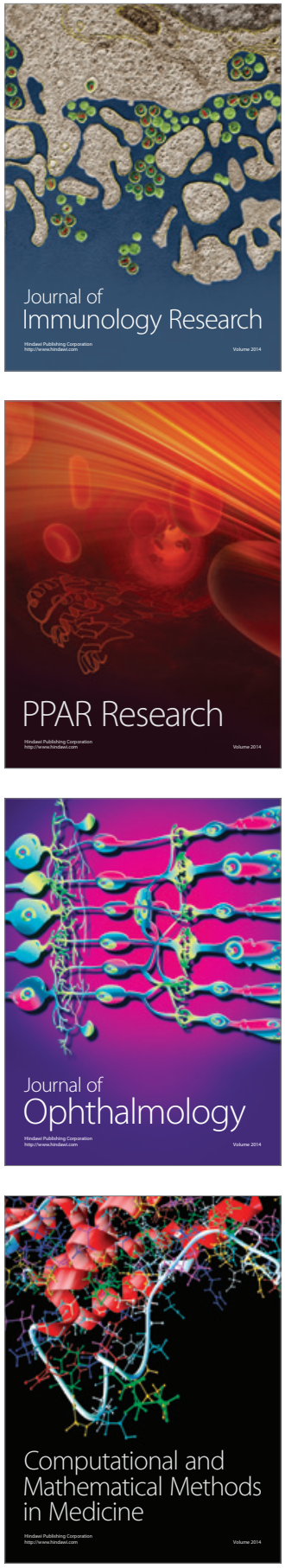

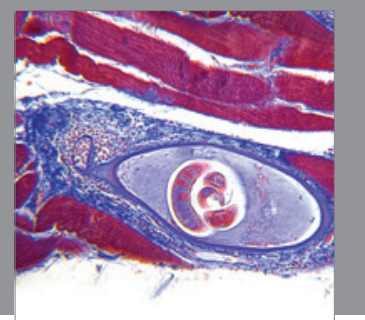

Gastroenterology

Research and Practice
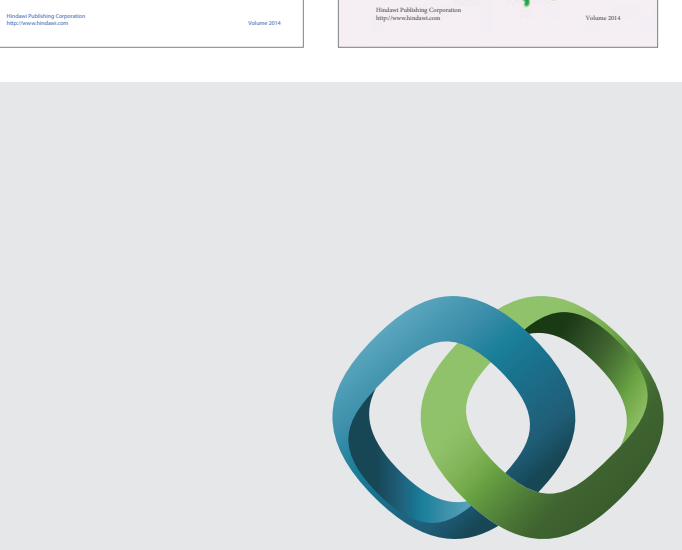

\section{Hindawi}

Submit your manuscripts at

http://www.hindawi.com
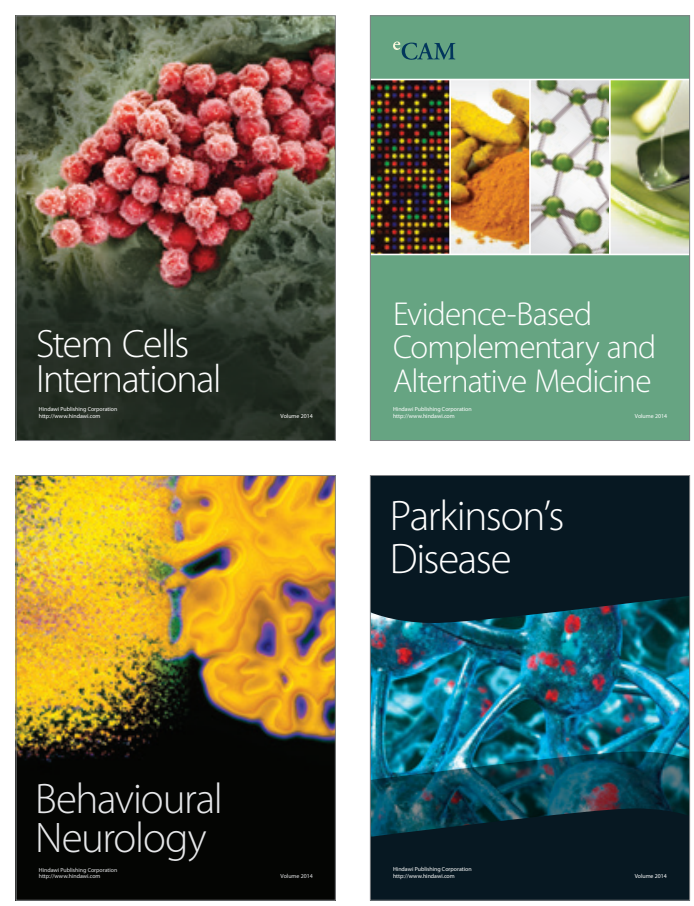

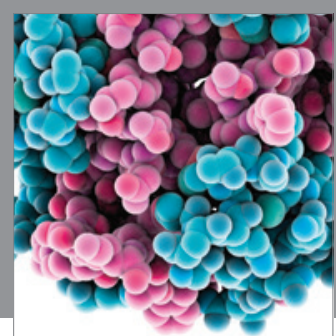

Journal of
Diabetes Research

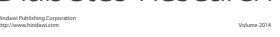

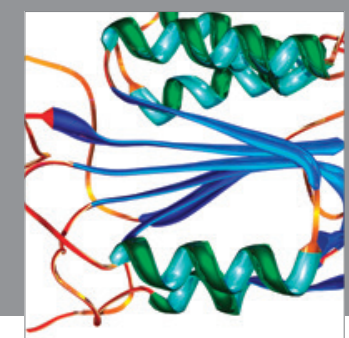

Disease Markers
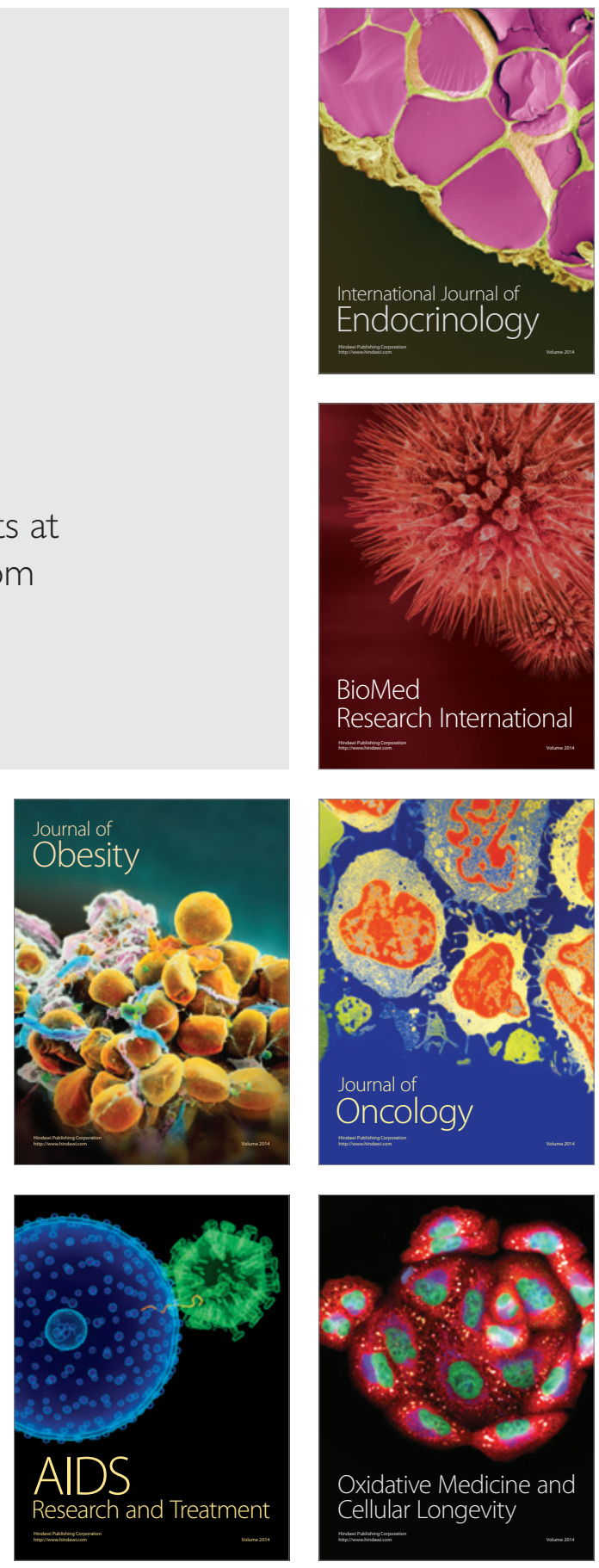